\title{
Research on Capability Evaluation System for Elevator Inspector
}

\author{
Kexi Fei ${ }^{1}$, Qinqiao Zhou ${ }^{1}$ and Kai Huang ${ }^{1, a}$ \\ ${ }^{1}$ Jiangsu Province Special Equipment Safety Supervision Inspection Institute, Nanjing, China
}

\begin{abstract}
This paper analyzes the current situation of elevator inspectors' training based on the QFD method. Then, an elevator inspector's capability evaluation system for elevator inspector is established. The evaluation index and the weight determination are presented in detail. The research can favor to assess the comprehensive capabilities of elevator inspectors, and provide a theoretical reference of appropriate training activities for elevator inspectors.
\end{abstract}

\section{Introduction}

With the rapid development of society and economy, the number of special equipment has grown at a rate of nearly $10 \%$ each year. Especially, the elevators closely related to daily work and life of people, have received much attention from society and the government ${ }^{[1]}$. Therefore, the establishment of a comprehensive evaluation system to identify and improve the elevator inspector's business level, so as to ensure the safe operation of elevator equipment, is of great significance and has become the development trend in this field ${ }^{[2]}$.

\section{QFD-based demand analysis of capability evaluation system for elevator inspector}

A QFD -based demand analysis is conducted to explore the relevance and sequencing of the evaluation needs. Through the progressive analysis at each level, the corresponding training objectives and training methods for elevator inspectors are established from the strategic and organizational levels, as well as the corresponding policy mechanisms ${ }^{[3]}$. The QFD-based requirements analysis flow chart is shown in Figure 1.

According to the demand analysis, it can be found that a complete and standard capability evaluation system needs to be established as an evaluation benchmark for elevator inspector performance ${ }^{[4]}$. At the same time, elevator inspectors need to be regularly assessed, evaluated, educated and trained to promote their comprehensive capabilities.

\section{Establishment of elevator inspector capability evaluation system}

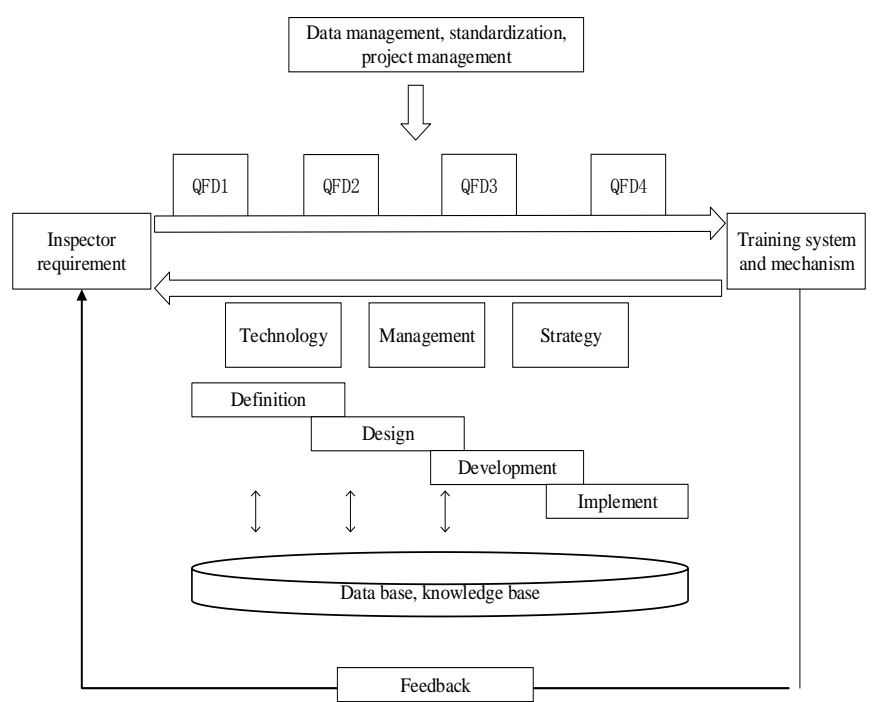

Figure 1. Flow chart of requirements analysis based on QFD.

(1) The establishment of elevator inspector skills evaluation criteria based on TSG Z8002-2013, GB/T 7024-2008

According to the results of the needs analysis, a set of evaluation criteria for the qualification of elevator inspectors from the aspects of qualification, inspection ability, learning and training, technical achievements, practical operation, and annual assessment shall be established ${ }^{[5]}$.

This standard is based on <elevators, escalators, moving sidewalk terminology $>$ (TSG Z8002-2013), $<$ special equipment inspection personnel assessment rules $>$ GB/T 7024-2008.The terminology definitions, evaluation indicators, calculation methods, and skill grading of skills are applicable to the skill evaluation of elevator in-service inspection personnel ${ }^{[6]}$. Details are as in Figure 2.

\footnotetext{
a Corresponding author: js_nj_hk@ $163 . c o m$
} 


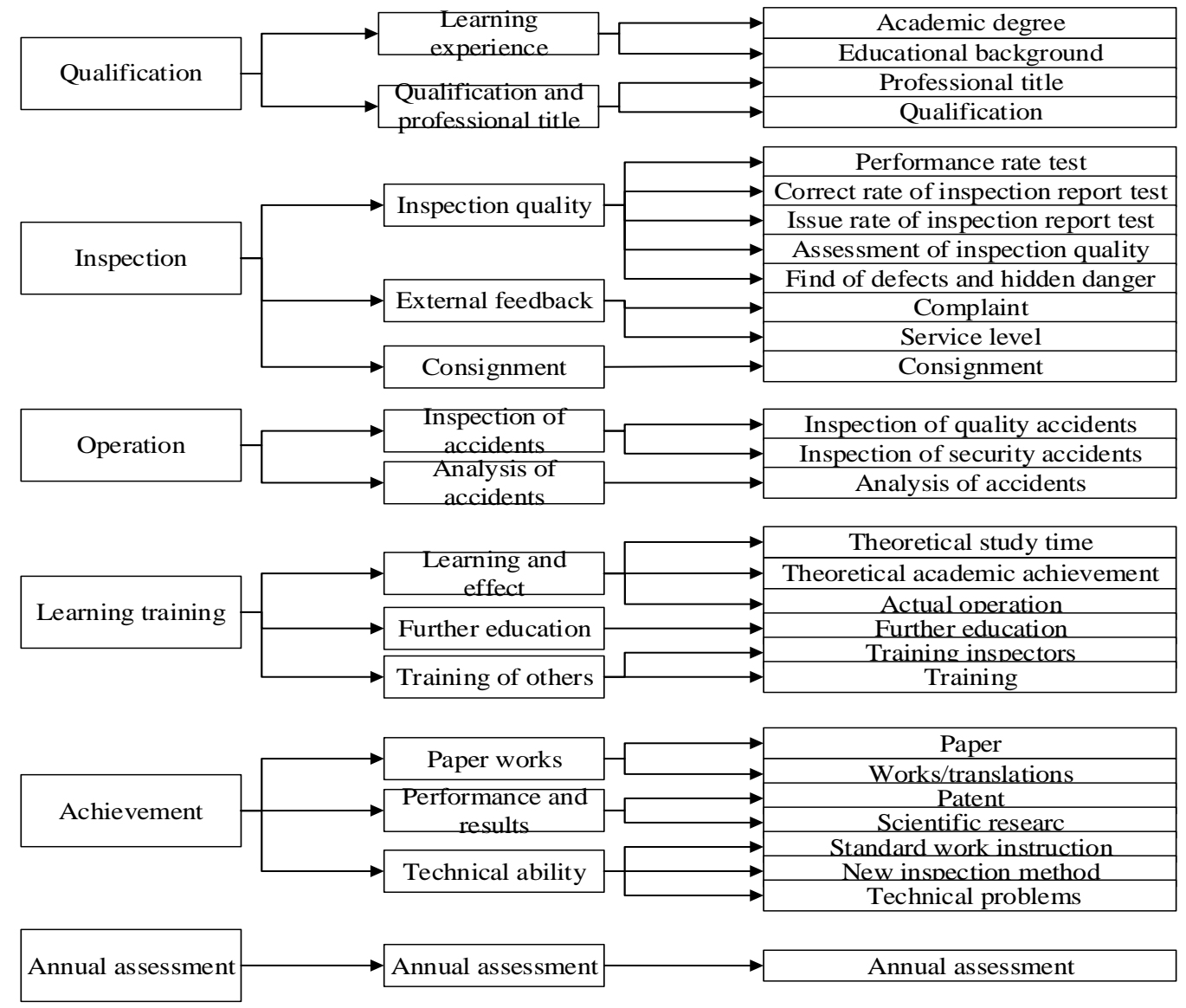

Figure 2. Elevator inspector evaluation standard system.

(2) Evaluation of elevator inspector ability based on fuzzy comprehensive evaluation

For the elevator inspector evaluation problem, most of the indicators are qualitative. By introducing the concept of membership degree, the boundary of membership function classification index is constructed to solve the problem. Finally, a fuzzy comprehensive evaluation can be performed ${ }^{[7]}$. The specific flow chart is shown in Figure 3.

Through the above process, a complete elevator inspector capability evaluation system has been established, which can comprehensively evaluate the elevator inspector's theoretical knowledge, methods, skills and other overall qualities ${ }^{[8]}$. The evaluation system established is shown in Table 1.

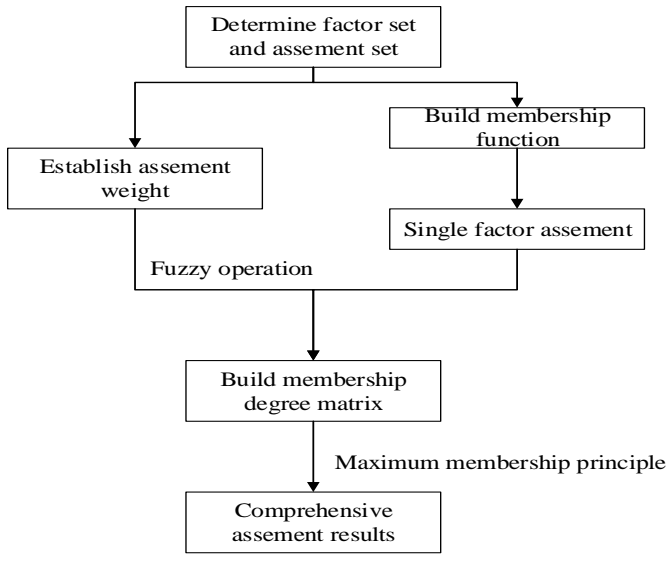

Figure 3. Fuzzy evaluation flowchart.

Table 1. Elevator Inspector Skills Evaluation System

\begin{tabular}{|c|c|c|c|c|c|}
\hline $\begin{array}{l}\text { One class } \\
\text { index }\end{array}$ & Weight & Two class index & Weight & Three class index & Weight \\
\hline \multirow{5}{*}{$\begin{array}{c}1 \\
\text { Qualification }\end{array}$} & \multirow{5}{*}{0.10} & \multirow{3}{*}{$\begin{array}{c}1.1 \\
\text { Learning experience }\end{array}$} & & Major & \\
\hline & & & \multirow{2}{*}{0.50} & Academic degree & 0.50 \\
\hline & & & & Educational background & 0.50 \\
\hline & & \multirow{2}{*}{$\begin{array}{l}1.2 \\
\text { Qualification and } \\
\text { professional title }\end{array}$} & \multirow{2}{*}{0.50} & Professional title & 0.50 \\
\hline & & & & Qualification & 0.50 \\
\hline
\end{tabular}

\footnotetext{
$\overline{{ }^{a} \text { Corresponding author: js_nj_hk@ }} 163 . c 0 m$
} 


\begin{tabular}{|c|c|c|c|c|c|}
\hline \multirow{8}{*}{$\begin{array}{c}2 \\
\text { Inspection } \\
\text { ability }\end{array}$} & \multirow{8}{*}{0.50} & \multirow{5}{*}{$\begin{array}{c}2.1 \\
\text { Inspection quality }\end{array}$} & \multirow{5}{*}{0.60} & Performance rate test & 0.05 \\
\hline & & & & Correct rate of inspection report test & 0.20 \\
\hline & & & & Issue rate of inspection report test & 0.15 \\
\hline & & & & Assessment of inspection quality & 0.30 \\
\hline & & & & Defects and hidden danger find & 0.30 \\
\hline & & \multirow{2}{*}{$\begin{array}{c}2.2 \\
\text { External feedback }\end{array}$} & \multirow{2}{*}{0.20} & Complaint & 0.50 \\
\hline & & & & Service level & 0.50 \\
\hline & & $\begin{array}{c}2.3 \\
\text { Consignment } \\
\end{array}$ & 0.20 & Consignment & 1.00 \\
\hline \multirow{3}{*}{$\begin{array}{c}3 \\
\text { Accident }\end{array}$} & \multirow{3}{*}{0.05} & & \multirow{2}{*}{0.50} & Quality accidents inspection & 0.50 \\
\hline & & Accidents inspection & & Security accidents inspection & 0.50 \\
\hline & & $\begin{array}{c}3.2 \\
\text { Accidents analysis }\end{array}$ & 0.50 & Accidents analysis & 1.00 \\
\hline \multirow{6}{*}{$\begin{array}{l}4 \\
\text { Learning and } \\
\text { Training }\end{array}$} & \multirow{6}{*}{0.20} & \multirow{3}{*}{$\begin{array}{c}4.1 \\
\text { Learning and effect }\end{array}$} & \multirow{3}{*}{0.50} & Theoretical study time & 0.30 \\
\hline & & & & Theoretical academic achievement & 0.30 \\
\hline & & & & Practical operation & 0.40 \\
\hline & & $\begin{array}{c}4.2 \\
\text { Further education } \\
\end{array}$ & 0.10 & Further education & 1.00 \\
\hline & & \multirow{2}{*}{$\begin{array}{l}4.3 \\
\text { Others training }\end{array}$} & \multirow{2}{*}{0.40} & Training inspectors & 0.50 \\
\hline & & & & Training & 0.50 \\
\hline \multirow{7}{*}{$\begin{array}{c}5 \\
\text { Technical } \\
\text { achievements }\end{array}$} & \multirow{7}{*}{0.10} & \multirow{2}{*}{$\begin{array}{c}5.1 \\
\text { Paper works }\end{array}$} & \multirow{2}{*}{0.30} & Paper & 0.50 \\
\hline & & & & Works/translations & 0.50 \\
\hline & & 5.2 & & Patent & 0.50 \\
\hline & & results & & Scientific research & 0.50 \\
\hline & & & & Standard work instruction & 0.40 \\
\hline & & $\begin{array}{c}5.3 \\
\text { Technical ability }\end{array}$ & 0.30 & New inspection method & 0.30 \\
\hline & & & & Major defects problem & 0.30 \\
\hline $\begin{array}{c}6 \\
\text { Annual } \\
\text { assessment }\end{array}$ & 0.05 & $\begin{array}{c}6.1 \\
\text { Annual assessment }\end{array}$ & 1.00 & Annual assessment & 1.00 \\
\hline
\end{tabular}

\section{Summary}

This study focuses on the construction of an elevator inspector capability assessment system. From system and actual needs analysis to standardized evaluation process management, an elevator inspector capacity evaluation system is established. The platform has the ability to assess the comprehensive capabilities of elevator inspectors and can favor the appropriate training activities for elevator inspectors.

\section{Acknowledgment}

This work was financially supported by the Program of Jiangsu Province Special Equipment Safety Supervision Inspection Institute $(\mathrm{KJ}(\mathrm{Y}) 2014032)$. The supports are gratefully acknowledged.

\section{References}

1. C. Hepler, Q.B. Belt, G. Mazur, Q.R. Belt, 18th Symposium on QFD, (2006) 
2. J.L. Jin, Y.M. Wei, J. Ding, Journal of Hydraulic Engineering, 144-147 (2004)

3. T. Zhang, Proceedings of the 2012 International Conference on Cybernetics and Informatics. (Springer New York, 1203-1210 2014). DOI 10.1007/978-1-4614-3872-4_154

4. D.U. Baojiang, Y. Wang, N.I. Jiawei, D.U. Qiuyu, Electronic Science \& Technology, (2017)

5. M.L., Siikonen, Planning and Control Models for Elevators in High-Rise Buildings, (1997)
6. M.D. Yang, C F. Chao, K.S. Huang, L.Y. Lu, Y.P. Chen, Automation in Construction 33, 48-60 (2013). DOI: 10.1016/j.autcon.2012.09.017

7. A. Kaneko, Collision test apparatus and collision test method (US, US7886574, 2011)

8. Y.M. Xiong, Y.M. Chen, Advanced Materials Research 204-210, 1041-1044 (2011). DOI: 10.4028/www.scientific.net/AMR.204-210.1041 